\title{
Quality or decomposer efficiency - which is most important in the temperature response of litter decomposition? A modelling study using the GLUE methodology
}

\author{
J. Å. M. Wetterstedt and G. I. Ågren \\ Department of Ecology, Swedish University of Agricultural Sciences, P.O. Box 7044, 75007 Uppsala, Sweden
}

Received: 3 November 2010 - Published in Biogeosciences Discuss.: 30 November 2010

Revised: 7 February 2011 - Accepted: 10 February 2011 - Published: 18 February 2011

\begin{abstract}
We still lack full mechanistic understanding of how the temperature history affects the future decomposition rate of litter and soil organic matter. To explore that, we used the GLUE modelling framework together with the Q-model and data from a needle litter incubation experiment to compare a differential temperature response of litter qualities to a temperature-dependent decomposer efficiency. The needle litter incubation was a full factorial design with the initial and final temperatures 5,15 and $25^{\circ} \mathrm{C}$. Samples were moved from the initial to the final temperature when approximately $12 \%$ of the initial carbon had been respired and the experiment terminated when an additional $12 \%$ had been lost. We used four variations of the Q-model; the litter was described as having one or two initial quality values and the decomposer efficiency was either fixed or allowed to vary with temperature. All variations were calibrated with good fits to the data subsets with equal initial and final temperatures. Evaluation against temperature shift subsets also showed good results, except just after the change in temperature where all variations predicted a smaller response than observed. The effects of having one or two initial litter quality values (fixed decomposer efficiency) on end-of-experiment litter quality and respiration were marginal. Letting decomposer efficiency vary with temperature resulted in a decrease in efficiency between 5 and $15^{\circ} \mathrm{C}$ but no change between 15 and $25^{\circ} \mathrm{C}$ and in substantial differences in litter quality at the end of the initial incubation in response to incubation temperature. The temperature response of decomposition through temperature dependent decomposer efficiency proved, therefore, to be more important than the differential response to different substrate qualities. These results suggests that it
\end{abstract}

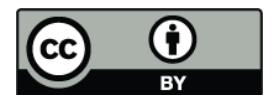

Correspondence to: G. I. Ågren (goran.agren@slu.se) may be important to consider other factors (e.g. microbial efficiency, changing substrate composition) than the temperature sensitivity coupled to substrate quality when evaluating effects of temperature changes on soil organic matter stability.

\section{Introduction}

In view of the expected future climatic change (Solomon, 2007) the temperature dependence of decomposition of litters and soil organic matter (SOM) has since long attracted much interest because a strong positive temperature dependence would create a strong positive climatic feedback. However, both in laboratory incubations and field studies, temperature history and not only current temperature have been shown to affect respiration rates, such that SOM with different temperature histories will have different decomposition rates at the same temperature. These effects can be short term or long term, and be a result of factors like substrate depletion, changes in decomposer community composition and abundance, and changes in quality composition (Kirschbaum, 2006). Quality changes have been challenged as a major factor because the temperature dependence of the rate of utilisation might not respond strongly enough to explain observations as there are several processes involved with sometimes counteracting temperature responses (Davidson and Janssens, 2006; Ågren and Wetterstedt, 2007). It is thus necessary to also consider the temperature dependence of the other factors regulating decomposition. Decomposer community composition and decomposer biomass are two important factors that may be affected by temperature. For example, it has been demonstrated (Devêvre and Horwáth, 2000; Steinweg et al., 2008) that the carbon use efficiency of decomposers decreases with

Published by Copernicus Publications on behalf of the European Geosciences Union. 
temperature, probably as a result of higher maintenance costs. Allison et al. (2010) suggest that the decomposer biomass should go down with increasing maintenance cost and as a result less extracellular enzymes are produced and decomposition slows down.

Most models, e.g. Century (Parton et al., 1987), G’Day (Comins and McMurtrie, 1993), RothC (Coleman and Jenkinson, 1995), Q (Ågren and Bosatta, 1998) dealing with soil organic carbon (SOC) conform to the same generic structure. SOC is described as consisting of carbon of different pools or qualities with three main processes driving the changes in SOC quantity and quality: (i) a decomposer community feeding on SOC at some rate (growth rate); (ii) when doing so, part of the carbon it uses is respired as carbon dioxide and part remains as SOC; we call the fraction remaining efficiency (decomposer efficiency); (iii) the fraction remaining undergoes changes in quality. We call this transfer between qualities (pools) dispersion, on average SOC increases in recalcitrance/decreases in quality with time.

To investigate how the factors quality and decomposer efficiency affect respiration at different temperatures (temperature response) we tested the Q-model (Bosatta and Ågren, 2003; Ågren and Bosatta, 1998) against an incubation experiment with needle litter (Wetterstedt et al., 2010). The Q-model was chosen because the fate of carbon and the decomposition processes are relatively easy to follow in it. The factors quality and decomposer efficiency are explored by modifying the model to have one or two initial litter qualities in combination with fixed or flexible (with regard to temperature) decomposer efficiency.

We have chosen to use the GLUE (Generalised Likelihood Uncertainty Estimation, Beven, 2006) framework for model calibration and evaluation. GLUE can be used as a modelling protocol and is well suited to give uncertainty estimations in model output. It also provides criteria for complete model rejection, i.e. the model structure needs to be changed if the model fails to predict empirical data well enough.

The main reason for choosing GLUE as opposed to a formal Bayesian approach was because it allowed us more freedom in specifying a likelihood function. The measurements are known to contain equipment-related errors, there is biological variation within the replicates, the Q-model is nonlinear, and the ever-present model structural error makes the identification of a formal error model to be used in a formal Bayesian approach problematic. Moreover, our main purpose is not to establish the value of the parameters to the best precision possible, but rather to explore qualitative effects of the parameters and the model. Using GLUE, it is often the case that quite different parameter sets give more or less equal good fits (equifinality). Within the framework it is easy and straightforward to use those sets in ensemble modeling. Even though the likelihood function is subjectively chosen it is easy to understand and communicate to a wider audience. The likelihood function is of lesser importance as long as it will help us find parameters that make the model predict measurements well. See also the discussion of the use of the GLUE methodology versus other formal Bayesian approaches (Mantovan and Todini, 2006; Beven et al., 2008).

\section{Materials and methods}

\subsection{The Q-model}

The Q-model describes litter or SOC as consisting of a continuous spectrum of carbon qualities or decomposabilities instead of being partitioned into a small number of discrete pools. The Q-model has certain advantages over discrete models. Firstly, there are analytical solutions, making it easier to understand and explain model behaviour. Approximate solutions, which are similar in their behaviour, are also available (Bosatta and Ågren, 2003). The approximate solutions are much less computationally demanding and are therefore preferred when doing large model runs, for example during calibration. They substitute the complete distribution of litter qualities in the exact solution with one average quality. Secondly, there are also fewer parameters in a continuous formulation, as opposed to pool models, and the model formulation enforces consistency between them (Bruun et al., 2010). Parameters estimated with the approximate solutions can also be used in the exact solution, possibly with some slight recalibration.

In the Q-model the growth rate $(u)$ of the decomposers depends on carbon quality $(q)$ and temperature $(T)$ as well as the base rate parameter $\left(u_{0}\right)$. The temperature response of the growth rate couples temperature and quality through an Arrhenius function with activation energy $\Delta G^{0}$ giving

$u(q, T)=u_{0} e^{-\frac{\left|\Delta G^{0}\right|}{q R T}}$

where $R$ is the gas constant (Bosatta and Ågren, 1999); we will for convenience allow $q>1$ in contrast to the restriction $0<q<1$ in the original derivation. Decomposer efficiency $(e)$ is set to be either temperature independent (fixed) or allowed to vary with temperature (flexible). In the latter case no specific temperature function has been assumed; the intention is instead to investigate the existence of a temperature dependence. Transfer of carbon to lower qualities, dispersion, is expressed through the parameter $\eta_{12}$, which is assumed to be temperature independent. Table 1 lists the parameters used in the model. When running the model with two initial litter qualities, the two $q_{0}$ 's will be selected from behavioural models (see below) where the qualities are somewhat separated. The reason we are using two initial $q_{0}$ 's in this experiment is to explore the effect of how the different temperature sensitivity of different $q_{0}$ 's translates into a differential quality evolution. Besides answering the question of which variations of the Q-model that can reproduce the observations, it is necessary to look at the consequences for the distribution of carbon qualities; when extrapolating 
Table 1. List of parameters. Parameter values are those corresponding to the highest LM. Range is the range used in the calibration procedure. Sampling was done uniformly for all parameters but $u_{0}$ which was sampled from a log-distribution. With two initial qualities the $q_{0}$ 's $\left(q_{0-1}\right.$ and $\left.q_{0-2}\right)$ were fixed from values obtained with one initial quality $\left(q_{0}\right)$. Fixed and Flex. in the table heading refer to fixed or flexible $e_{0}$. Initial parameter range was greater to find suitable parameter space.

\begin{tabular}{|c|c|c|c|c|c|c|}
\hline \multirow[b]{2}{*}{ Parameter } & \multirow[b]{2}{*}{ Range } & \multicolumn{2}{|c|}{ One $q_{0}$} & \multicolumn{2}{|c|}{ Two $q_{0}$} & \multirow[b]{2}{*}{ Comment } \\
\hline & & Fixed & Flex. & Fixed & Flex. & \\
\hline$q_{0}$ & $1.5-4.5$ & 2.50 & 2.5 & - & - & Initial litter quality (unit-less) \\
\hline$q_{0-1}$ & fixed & - & 1.80 & & 1.80 & 1st initial litter quality in the two $q_{0}$ version \\
\hline$q_{0-2}$ & fixed & - & 2.50 & & 2.50 & 2nd initial litter quality in the two $q_{0}$ version \\
\hline$\Delta G^{0}$ & a & 127 & 93 & 127 & 99.5 & Gibbs' free energy $(\mathrm{kJ})$ \\
\hline$e_{0}$ & $0.15-0.40$ & 0.377 & - & 0.220 & - & $\begin{array}{l}\text { Decomposer efficiency in the } \\
\text { fixed version (unit-less) }\end{array}$ \\
\hline$e_{0-5}$ & $-:-$ & - & 0.369 & - & 0.393 & $e_{0}$ at $5^{\circ} \mathrm{C}$ in the flexible version \\
\hline$e_{0-15}$ & $-:-$ & - & 0.327 & - & 0.336 & $e_{0}$ at $15^{\circ} \mathrm{C}$ in the flexible version \\
\hline$e_{0-25}$ & $-:-$ & - & 0.321 & - & 0.330 & $e_{0}$ at $25^{\circ} \mathrm{C}$ in the flexible version \\
\hline$u_{0}$ & $10^{7}-10^{9}$ & $7.63 \times 10^{7}$ & $2.46 \times 10^{7}$ & $65.7 \times 10^{7}$ & $1.21 \times 10^{7}$ & $\begin{array}{l}\text { Decomposer growth rate regulating } \\
\text { parameter }\left(\mathrm{gC}(\mathrm{g} \mathrm{C})^{-1} \mathrm{~d}^{-1}\right)\end{array}$ \\
\hline$\eta_{12}$ & $0.1-0.45$ & 0.120 & 0.199 & 0.0663 & 0.0476 & $\begin{array}{l}\text { Rate of quality decrease, } \\
\text { approximate solution (unit-less) }\end{array}$ \\
\hline$Q_{10}$ & $1.4-4.0$ & & & & & $\begin{array}{l}\text { Only used to create } \\
\text { plausible parameter range for } \Delta G^{0}\end{array}$ \\
\hline
\end{tabular}

a For $\Delta G^{0}$ the range is $q_{0} R \frac{T_{5} T_{15}}{10}\left[-\log \left[Q_{10 \max }\right] \ldots-\log \left[Q_{10 \min }\right]\right]$.

from this short-term experiment to long-term carbon storage differences in quality distributions become important.

Four versions of the model were run with combinations of one or two initial qualities combined with fixed or flexible decomposer efficiencies. When using two initial qualities, one quality was chosen as the best one found when using only one quality and the other one was set to a lower value estimated to give a reasonable difference; the sensitivity to this choice was also tested. The initial amount of carbon was partitioned equally between the two qualities. When estimating parameters within GLUE we have used the approximate version of Model III as defined in Bosatta and Ågren (2003). The best parameter set has then been used in the exact solution to calculate quality distributions.

\subsection{Observational data}

We have chosen to use the spruce (Picea abies) needle litter data from the temperature experiment by Wetterstedt et al. (2010) (see Figs. 1 and 2). The data consists of time series (four replicates) of litter respiration rates at different temperatures. In some time series the samples have been shifted from one temperature to another when approximately $12 \%$ of the initial carbon had been respired. We will denote temperature combinations as initial temperature + final temperature, e.g. $5+15^{\circ} \mathrm{C}$, meaning that the sample was first exposed to $5^{\circ} \mathrm{C}$ and then $15^{\circ} \mathrm{C}$. The data used for calibration were from needles stored at three temperatures without shifts in temperature $\left(5+5^{\circ} \mathrm{C}, 19\right.$ data points; $15+15^{\circ} \mathrm{C}, 14$ data points; and $25+25^{\circ} \mathrm{C}, 16$ data points). To reduce the variability in data between measurement points, we used a running mean of three consecutive points to smooth the curve (the first and last points were averaged from two points). We also normalised the variance at each measurement point by averaging over the whole measurement period for each temperature, i.e. the variances used when calibrating the model are

$$
\bar{O}_{i} \sum_{i} \frac{\operatorname{Var}\left(\bar{O}_{i}\right)}{\bar{O}_{i}} / n,
$$

where $O_{i}$ is the observation at point $i$ and $n$ is the number of points in the measurement series. These steps were taken to obtain a more robust calibration process. However, when the calibrated models are evaluated using the least square method, $R^{2}$ are calculated with respect to non-smoothed data (Figs. 1 and 2).

\subsection{GLUE}

The GLUE methodology introduced by Beven and Binley (1992) is a framework for calibrating and using models in predictions. It includes criteria and methods for model rejection and sensitivity analysis of model parameters. A "model" in GLUE terminology is the combination of the "model structure", e.g. the Q-model as opposed to some other model, and the parameter values used to run the model. A "behavioural model" is a model that can simulate real data "good enough". It follows that a non-behavioural model 

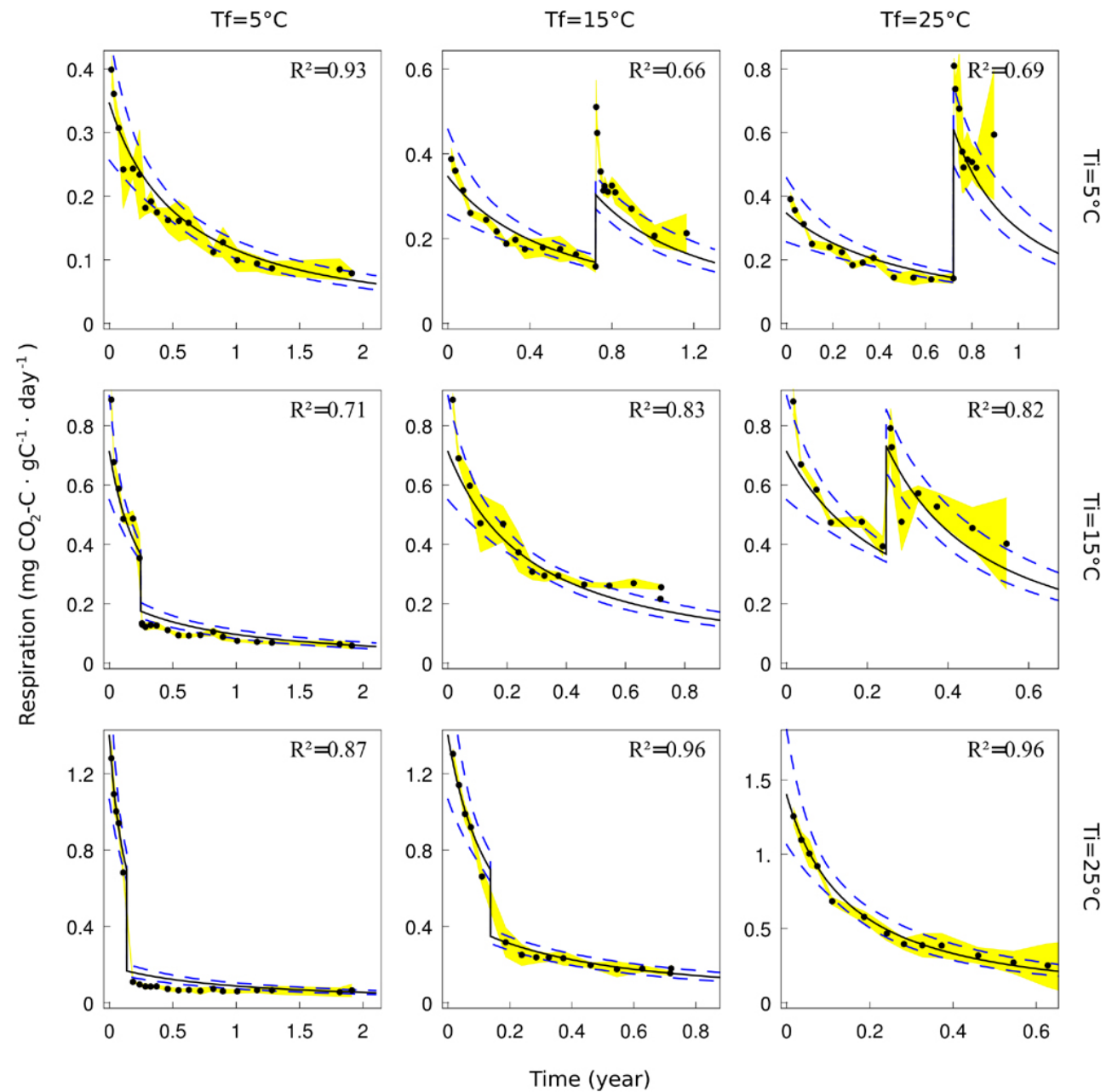

Fig. 1. Model predictions of respiration rates for the one initial quality, fixed decomposer efficiency model and observed respiration rates for all combinations of initial $\left(T_{\mathrm{i}}\right)$ and final $\left(T_{\mathrm{f}}\right)$ temperatures $\left(5^{\circ} \mathrm{C}, 15^{\circ} \mathrm{C}\right.$, and $25^{\circ} \mathrm{C}$.) Weighted ensemble run predictions (solid black line) with $\mathrm{max} / \mathrm{min}$ curves (blue dashed lines) for the behavioural parameter sets. The yellow fields show $95 \%$ error bounds around measured data points (dots). Least square $\mathrm{R}^{2}$-values are shown in top right corner of each sub-graph.

should not be used to forecast data; instead, it would need better parameterisation or a change in model structure. In this text we will, however, use the term "model" with the meaning "model structure".

The GLUE methodology is particularly useful in the field of environmental modelling in which the errors involved in measurement data may be unclear and where the response surface of the "goal function" or likelihood measure (LM) is flat and likely to contain many local optima (cf. Hyvönen et al., 2005). The GLUE methodology also acknowledges that more optima will be found with a more extensive search in the parameter space. Since it is likely that these optima would move with already small differences in measurement data (measurement errors), it is not meaningful to only search for a global optimum.

The use of GLUE includes the following steps (Beven, 2009):
1. Likelihood measure: decide on an informal (or formal) likelihood measure or measures (LM) for use in evaluating each model run, including the rejection criteria, which for a non-behavioural model run will be given a likelihood of zero. Ideally this should be done before running the model, taking into account possible input and observational errors: since calibration data contain means as well as standard deviations, we used a triangular shaped likelihood measure:

$l\left(M, O_{\text {min }}, O_{\text {mean }}, O_{\max }\right)=\left\{\begin{array}{l}\frac{M-O_{\min }}{O_{\operatorname{mean}}-O_{\min }} M \leq O_{\text {mean }} \\ \frac{O_{\max }-M}{O_{\max }-O_{\text {mean }}} M>O_{\text {mean }}\end{array}\right\}$

If model output equals the average measured value $\left(O_{\text {mean }}\right)$ the function returns 1 , at \pm 1.96 standard deviations $\left(O_{\max }, O_{\min }\right)$ zero, and negative values when model output deviates more from the observed mean 

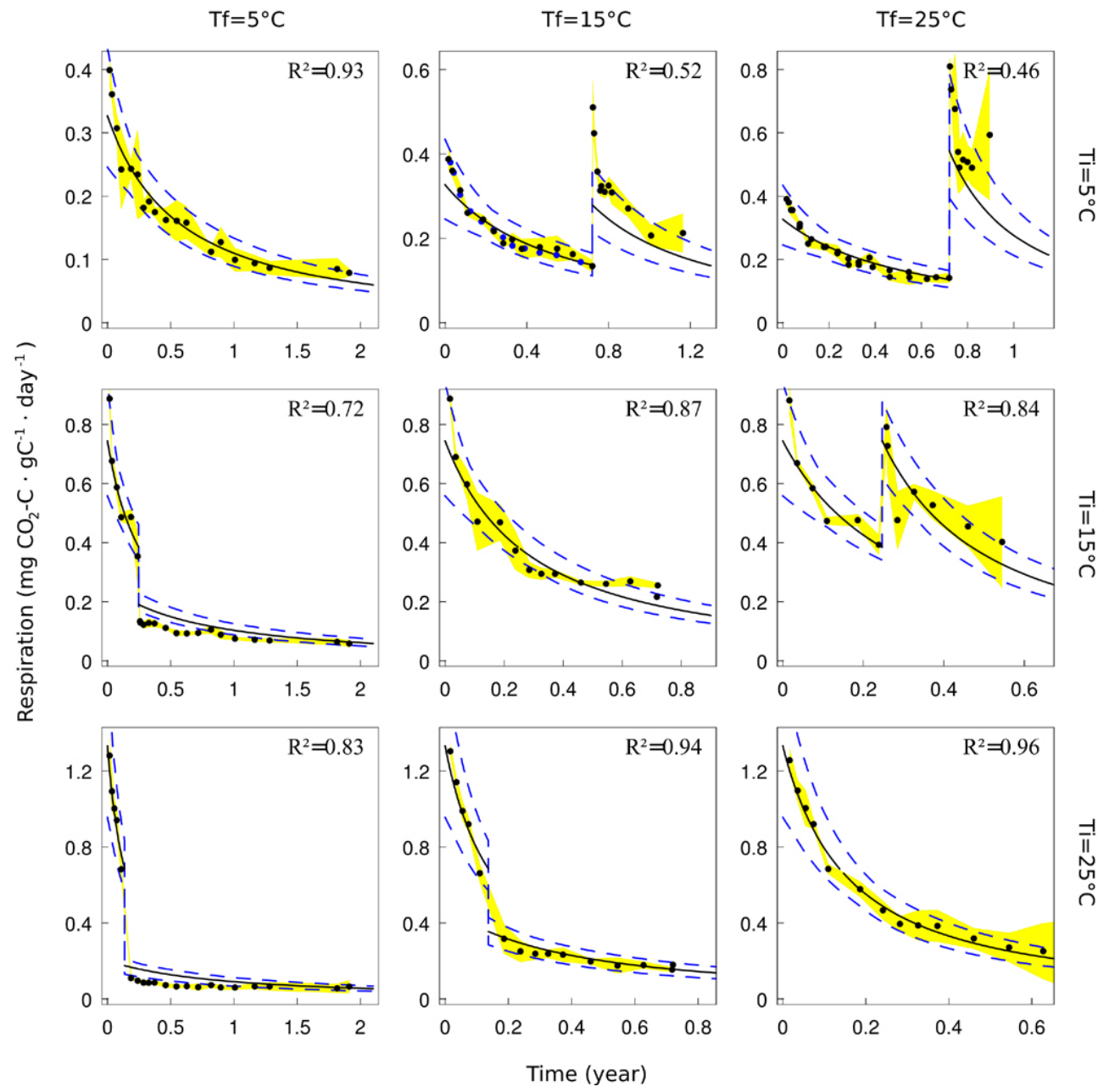

Fig. 2. Model predictions of respiration rates for the one initial quality, flexible decomposer efficiency model and observed respiration rates for all combinations of initial $\left(T_{\mathrm{i}}\right)$ and final $\left(T_{\mathrm{f}}\right)$ temperatures $\left(5^{\circ} \mathrm{C}, 15^{\circ} \mathrm{C}\right.$, and $25^{\circ} \mathrm{C}$.) Weighted ensemble run predictions (solid black line) with max/min curves (blue dashed lines) for the behavioural parameter sets. The yellow fields show $95 \%$ error bounds around measured data points (dots). Least square $\mathrm{R}^{2}$-values are shown in top right corner of each sub-graph.

value. These values were summed for each temperature and divided by the number of observed values, $n_{O, T}$ and then averaged over the three temperatures

$$
\begin{aligned}
& \operatorname{LM}(M(\Theta), O) \\
& =\sum_{T=5,15,25^{\circ} \mathrm{C}}\left(\sum_{O_{T}} \frac{l\left(M_{O, T}, O_{\min , T}, O_{\text {mean }, T}, O_{\max , T}\right)}{n_{O, T}}\right) / 3 .
\end{aligned}
$$

$\Theta$ stands for the parameter set used. This choice of likelihood measure gives equal weight to the different temperature series. It takes also advantage of the variability in the observed data and is less influenced than the least square method by outliers. Ideally, the model with the parameters in question, should predict all observed data points within their $95 \%$ error bounds; i.e. for all $\mathrm{LM}\left(M, O_{\min }, O_{\text {mean }}, O_{\max }\right)$ observations. How- ever, this turned out not to be feasible, why have chosen $\mathrm{LM}>0$ as criteria for a behavioural model.

2. Model parameters: decide which model parameters and input variables are to be considered uncertain: all model parameters were considered uncertain (Table 1).

3. Parameter distributions: decide on prior distributions from which the uncertain parameters and variables can be sampled: we have chosen uniform initial distributions for all parameters except $u_{0}$, for which a logarithmic one was used (Table 1). To further narrow the sampling space, initial sample runs were made to localise parts of the parameter space that were more likely to generate good fits.

4. Random realisations of the model: decide on a method to generate random realisations of models consistent 

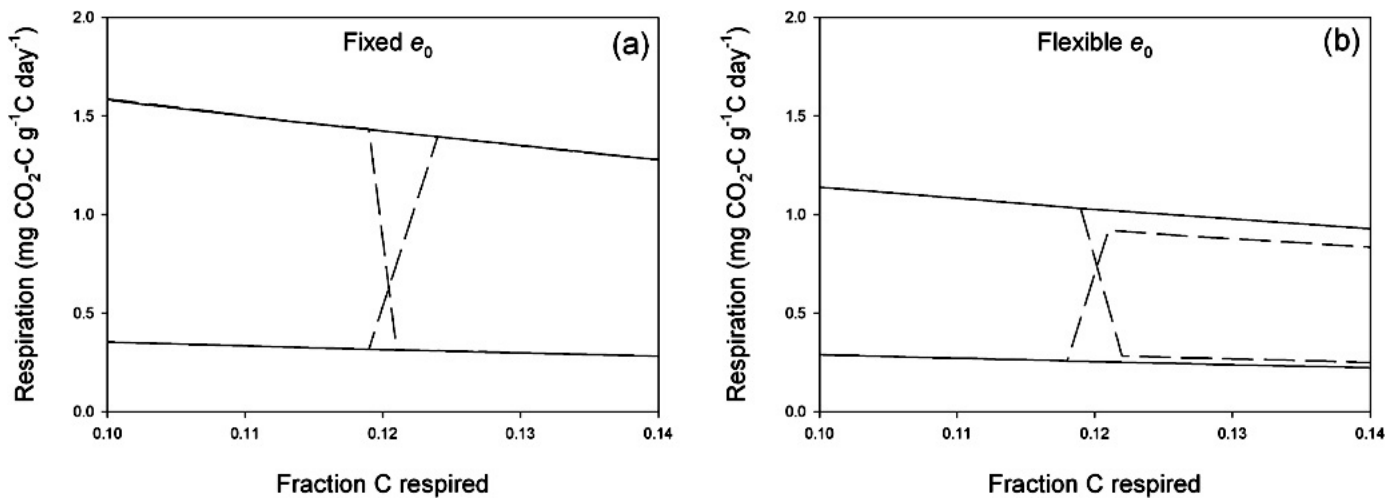

Fig. 3. Respiration rates of samples as a function of time, predicted by the flexible and fixed decomposer efficiency models with constant temperatures (solid lines) and with a switch between $5{ }^{\circ} \mathrm{C}$ and $25^{\circ} \mathrm{C}$ at $12 \%$ respired carbon (broken lines) using the parameter set with the highest LM (Table 1). For clarity, the transitions between temperatures are slightly displaced from exactly $12 \%$. (a) Fixed $e_{0}$. (b) Flexible $e_{0}$. Note, this simulation does not give the same result as the weighted ensemble predictions (Figs. 2 and 3).

with the assumptions in steps 1 and 2: twenty thousand parameter sets were drawn from uniform distributions for all parameters except $u_{0}$, which was drawn from a log-distribution, (Table 1) and used as initial points in the Simulated annealing algorithm (Mathematica 7.0.0 Ubuntu/Linux). This procedure results in only one "optimum" set of parameters. The procedure was therefore repeated 28000 times and the optimum sets together with their resulting likelihood values were stored. Calibration was made simultaneously against samples that had been kept at $5+5^{\circ} \mathrm{C}, 15+15^{\circ} \mathrm{C}$ and $25+25^{\circ} \mathrm{C}$.

\section{Dotty plots}

There exist a number of methods to assess sensitivity in nonlinear models. The method most often used within the GLUE framework is to make a scatter-plot/dotty plot of each parameter (on the $\mathrm{x}$-axis) versus the likelihood measure (y-axis) obtained during the calibration/conditioning process. From the resulting swarm of points, one can find trends showing for example that certain parameters are present in only a short interval of the initially sampled points, whereas others have a uniform density along the $\mathrm{x}$-axis. If only a small segment of the initially sampled parameter space is found among the behavioural model runs, restricting that parameter to a smaller range will probably improve the number of behavioural model runs. On the contrary, if behavioural runs are equally distributed along the parameter axis, extending the parameter range might disclose/unravel areas of the parameter space which are more likely to prove behavioural.

\subsection{Using the model}

Behavioural parameter sets are used in ensemble runs to generate a mean output value and likely error bounds. An ensemble run is obtained when running the same model with many parameter sets (as is the case in this article) or run- ning different models to obtain a distribution of results. The likelihood measure, LM, or any other performance measure, can then be used to weigh the different outputs to create a weighted mean. Error bounds can be generated from the max and $\min$ from the model runs, or at any preferred significance level obtained from a cumulative density curve. In this article we will simply use $\max$ and $\min$ of the selected models as bounds.

\section{Results}

\subsection{One initial quality, fixed decomposer efficiency}

With one initial quality $\left(q_{0}\right)$ and fixed decomposer efficiency $\left(e_{0}\right)$ the dotty plots (data not shown) showed more or less evenly distributed LM's, except for $u_{0}$ for which fits tended to be better with increasing $u_{0}$. This indicates that the upper boundary for $u_{0}$ might have been too small. The generally even distribution of all parameters means that, within the used ranges, different parameters compensate for each other, making the model rather insensitive to changes in single parameters. We got 257 parameter sets that were behavioural. The best fit yielded a LM of 0.243 , and was within boundaries at 37 out of 48 data points in the calibration set (Fig. 1).

When validated against experiments with shifts in temperature, the model follows the data well during the initial temperature phase; this is not surprising because it was calibrated on similar data (Fig. 1). During the final incubation after a temperature increase, the model underestimates the increase in respiration during the first days when going from $5^{\circ} \mathrm{C}$ to $15^{\circ} \mathrm{C}$ or $25^{\circ} \mathrm{C}$. When shifting downwards in temperature the model predicts initially slightly higher values than observed.

With fixed decomposer efficiency, temperature history has negligible effect on future respiration rates. The respiration at the final temperature after the shift for the $5+25^{\circ} \mathrm{C}$ treatment is the same as the respiration after $12 \% \mathrm{C}$ loss in 

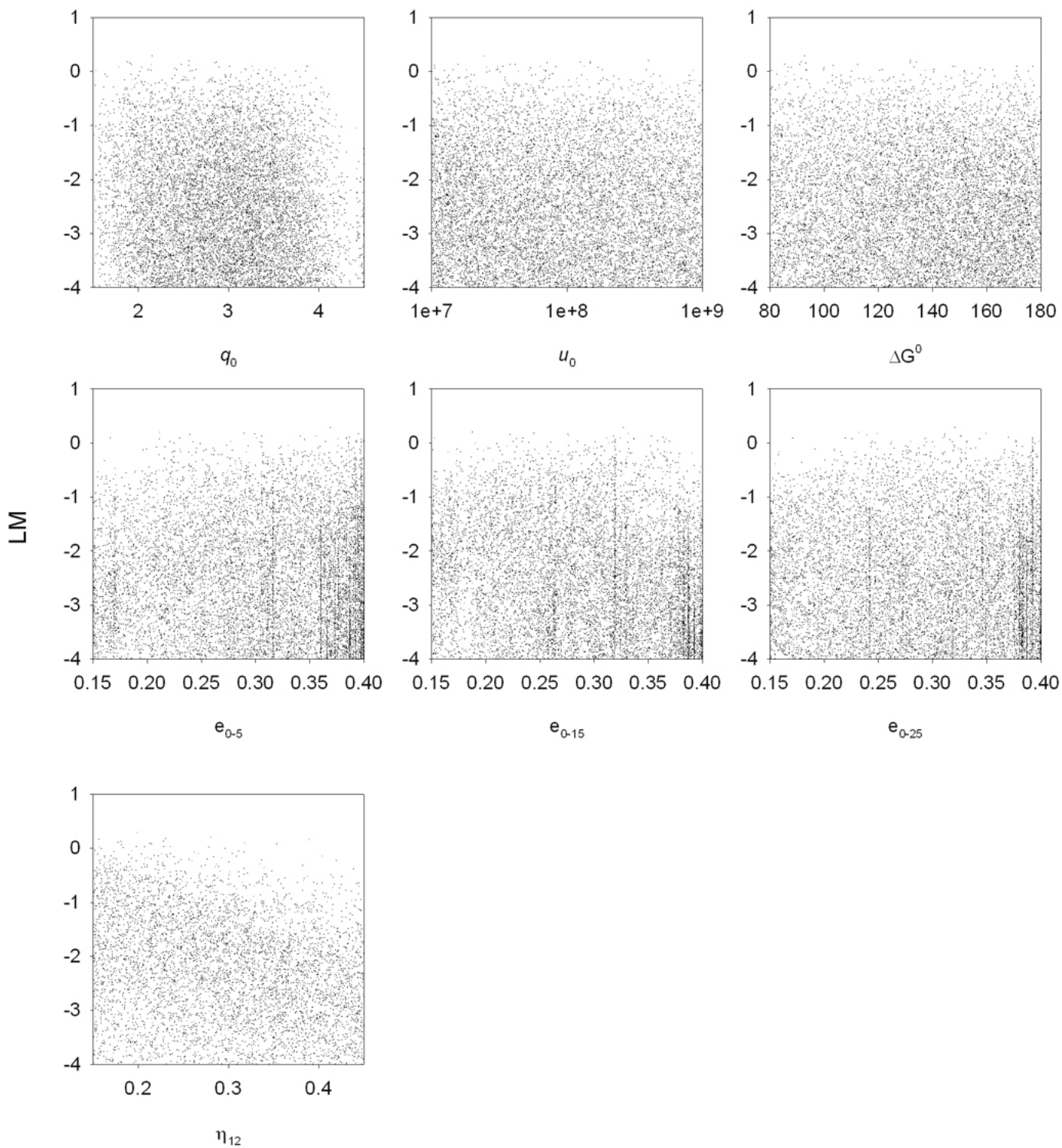

Fig. 4. Dotty plots for the one initial quality, flexible decomposer efficiency. Each dot represents one model run. The likelihood measure, LM, or "goodness of fit", is plotted against parameter values. The x-scales cover the allowed ranges of the parameters. Parameter sets resulting in a $L M>0$ where used in ensemble simulation runs.

the $25+25^{\circ} \mathrm{C}$ treatment (Fig. 3a). The same holds for the $25+5^{\circ} \mathrm{C}$ and $5+5^{\circ} \mathrm{C}$ treatment.

\subsection{One initial quality, flexible decomposer efficiency}

With one initial quality $\left(q_{0}\right)$ and flexible decomposer efficiency $\left(e_{0}\right)$ there are few points in the dotty plots at the extremes of the $\mathrm{x}$-axis for $q_{0}$, meaning that high and low $q_{0}$ were unlikely to give good fits. $u_{0}, \Delta G^{0}$ and $e_{0}$ 's are fairly evenly distributed. $\eta_{12}$ is skewed towards the lower end of the spectrum (Fig. 4). The best fit yielded a LM of 0.284 , and was within boundaries at 37 out of 48 points (Fig. 2). 33 sets were found behavioural.
The model with flexible decomposer efficiency fits the data slightly better than the fixed decomposer efficiency version when validated against the experiment with temperature shifts, as well as bracketing more of the data points due to the wider uncertainty bounds (Fig. 2). When going down in temperature, the model seems to over-shoot slightly, at least initially $\left(15+5^{\circ} \mathrm{C}, 25+5^{\circ} \mathrm{C}\right)$ and when going up $\left(5+25^{\circ} \mathrm{C}\right.$ and possibly $15+25^{\circ} \mathrm{C}$ ) the model misses the initial respiratory peak.

To search for trends in how $e_{0}$ varied with temperature we reran the simulations with the one $q_{0}$ flexible $e_{0}$ model to obtain a larger number (160) of behavioural parameter sets $(\mathrm{LM}>0)$. Decomposer efficiencies were plotted in pairs, i.e. 

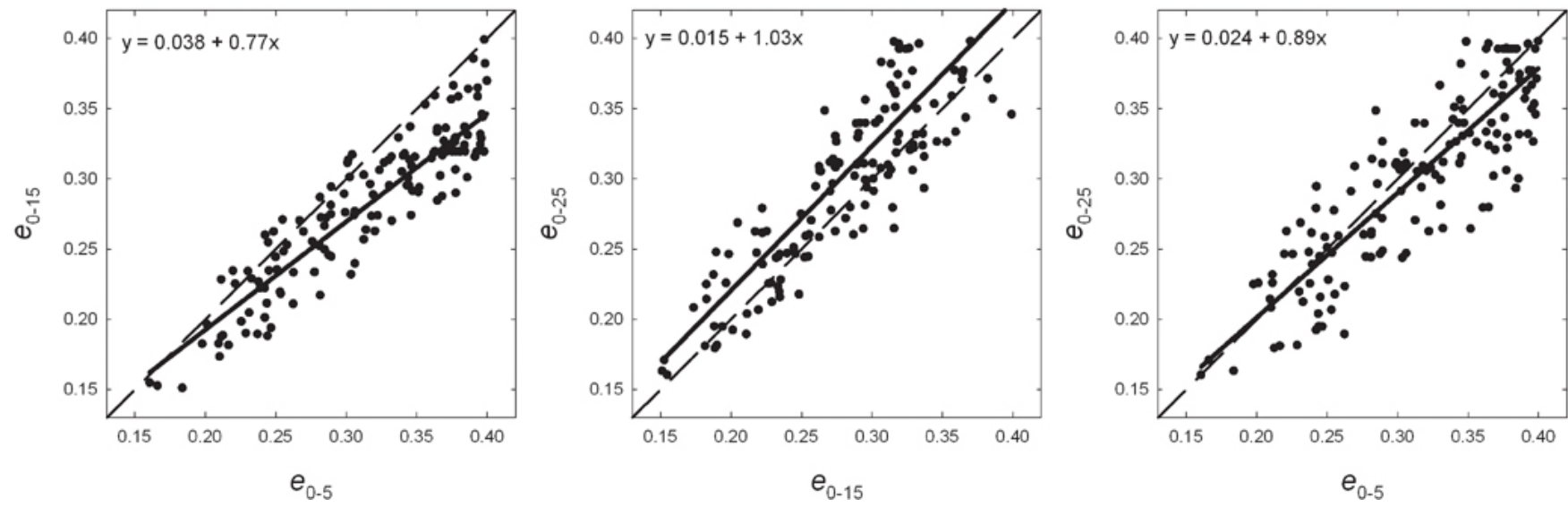

Fig. 5. Correlations between efficiencies at $5^{\circ} \mathrm{C}, 15^{\circ} \mathrm{C}$, and $25^{\circ} \mathrm{C}$ from behavioural parameter sets in the one initial quality, flexible decomposer efficiency model. Solid line: linear regression of data. Broken line: 1:1 line.

$e_{0-5}$ versus $e_{0-15}, e_{0-15}$ versus $e_{0-25}$, and $e_{0-5}$ versus $e_{0-25}$ (Fig. 5). The plots show that the $e_{0}$ 's are highly correlated. Average $e_{0}$ decreased by 0.03 units when going from $5{ }^{\circ} \mathrm{C}$ to $15^{\circ} \mathrm{C}$ (left), increased by 0.02 when going from $15^{\circ} \mathrm{C}$ to $25^{\circ} \mathrm{C}$ (middle), resulting in an overall decrease of 0.01 from $5^{\circ} \mathrm{C}$ to $25^{\circ} \mathrm{C}$ (right).

With temperature dependent decomposer efficiency, respiration responded strongly to temperature history. For example, the sample initially at $25^{\circ} \mathrm{C}$ respired substantially more than the one initially at $5^{\circ} \mathrm{C}$ when both were at $25^{\circ} \mathrm{C}$. Similarly, the sample initially at $25^{\circ} \mathrm{C}$ respired more than the one initially at $5^{\circ} \mathrm{C}$ when both are at $5^{\circ} \mathrm{C}$ (Fig. 3b).

\subsection{Two initial qualities, fixed and flexible decomposer efficiency}

In the model runs with two initial qualities, the contribution (both at time 0 and at $12 \%$ carbon loss) of the lower quality $\left(q_{0-1}\right)$ to respiration is much lower $(1 / 4700$ and $1 / 2700$ at $5^{\circ} \mathrm{C}$ and $25^{\circ} \mathrm{C}$, respectively) than the respiration of the higher quality $\left(q_{0-2}\right)$. Therefore the respiration from the high quality totally dominated the respiration and the model behaved qualitatively the same as with a single initial quality but with different "optimal" parameters.

\section{Discussion}

\subsection{Model behaviour}

The calibration data showed considerable variation in the variability between days. Also, the respiration did not always decrease monotonically as expected. We do not know whether this variability in input data comes from short-time biological variation or from measurement errors. We had, therefore, to relax the condition that, for each behavioural parameter set, predictions should be within error bounds for all points in each temperature series. Despite that, calibration to the constant temperature subsets worked well with $R^{2}$ values in the range of $0.83-0.96$. However, even though the ensemble runs mostly covered all calibration points, at $5^{\circ} \mathrm{C}$ and $15^{\circ} \mathrm{C}$ the data points might have a more concave pattern than what the model can predict (Figs. 1 and 2 at $5+5^{\circ} \mathrm{C}$, $\left.15+15^{\circ} \mathrm{C}\right)$. When the model is validated against the temperature shift experiments, experiments tend to respond more strongly just at the temperature shift than the model.

\subsection{Choice of likelihood measure (LM)}

Our choice of likelihood measure, LM, is subjective. Ultimately, the objective should be to acquire parameters "useful in model prediction" (Beven, 2009, p. 124), and the LM should be chosen to help in doing so. One way of interpreting "useful in model prediction" is that the model should be able to bracket our observations, which it did in most of the cases (Figs. 1 and 2). Since the primary objective of this paper is not to model decomposition in general, but rather to highlight the effects on quality composition and respiration rates of a temperature-dependent efficiency and the coupling of quality with temperature, the likelihood measure used is of lesser importance; see Beven (2009, p. 165) for further discussion of choice of likelihood measure.

\subsection{Mechanisms for temperature history to influence current respiration}

\subsubsection{One or several initial qualities}

We have considered two main ways in which temperature history can affect current respiration rates. The first is that different qualities have different temperature dependencies, which should lead to a difference in quality composition at different temperatures and equal carbon loss (for a more detailed discussion, see Wetterstedt et al., 2010). However, 

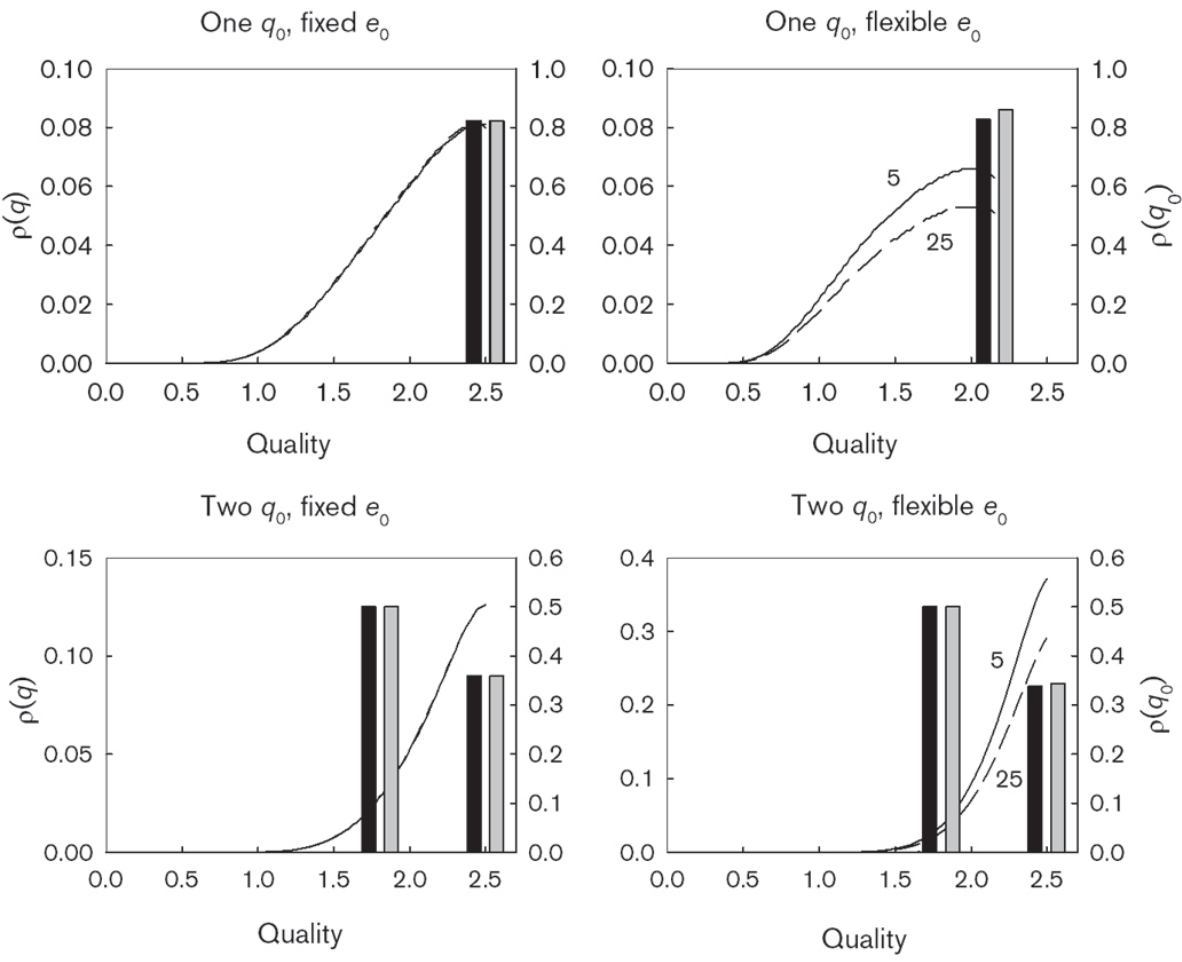

Fig. 6. Distributions of qualities for combinations of one or two initial qualities and fixed or flexible decomposer efficiency $\left(e_{0}\right)$ when $12 \%$ carbon has been lost for samples incubated at $5{ }^{\circ} \mathrm{C}$ or $25^{\circ} \mathrm{C}$. Solid lines and black bars are for samples at $5{ }^{\circ} \mathrm{C}$. Dashed lines and grey bars are for samples at $25^{\circ} \mathrm{C}$. The bars have been shifted slightly leftwards and rightwards from their value to visually separate them. Bars show the amount of carbon that has not been used by decomposers so far (remaining at the initial qualities). The lines show the distributions of carbon that the decomposers have converted into new qualities. With flexible decomposer efficiency, more carbon has been converted (lines) and less remains at the initial quality (bar) at the higher temperature. With two initial qualities the losses have essentially only occurred from the highest quality. Note that the sums of the bar(s) and the areas under the corresponding temperature curves are all equal, $88 \%$ of initial carbon.

with our choice of two different initial qualities $\left(q_{0-1}=1.80\right.$, $\left.q_{0-2}=2.50\right)$, the lower quality decomposed at only about $1 / 4700$ at $5{ }^{\circ} \mathrm{C}$ respectively $1 / 2700$ at $25^{\circ} \mathrm{C}$ of the rate of the higher one. Together with the relatively small difference in temperature sensitivity between the two $q_{0}$ 's (fixed $e_{0}$, two $q_{0}$ model: $Q_{10-1}=2.9, Q_{10-2}=2.1$; flexible $e_{0}$, two $q_{0}$ model: $\left.Q_{10-1}=2.9, Q_{10-2}=2.3\right)$ this did not in this short-term experiment translate into a sufficiently large quality evolution between the temperatures; it is essentially only the highest quality that decomposes. Choosing a larger $q_{0-1}$ resulted in more use also of the lower quality, but at the expense of a smaller difference in $Q_{10}$ between the two qualities. However, whatever $q_{0-1}$ is chosen, the effect on the temperature response is small.

\subsubsection{Fixed or flexible decomposer efficiency}

The second mechanism, varying decomposer efficiency $\left(e_{0}\right)$ with temperature, resulted in a clear effect on quality distributions and thus temperature sensitivity and respiration rates (Figs. 3 and 6). The reason for this is two-fold. Most importantly, with higher efficiency, when carbon is taken from the initial quality, a smaller part is lost by respiration and a larger part is converted to lower qualities. Thus, to obtain the same mass loss more of the initial quality has to be processed. Secondly, the dispersed carbon will for the same reason persist for longer which means that yet more initial carbon needs to be processed before reaching the same cumulative respiration as at the lower decomposer efficiency. As a result at equal mass losses, the higher efficiency produces a lower quality.

The flexible model is better fitted to the initial more rapid increase at the beginning of the experiment as well as after the shift in temperature. Having a temperature dependent $e_{0}$ also leads to a model that simulates differences in respiration rates at the same final temperature from samples of different initial incubation temperatures (Fig. 3). Surprisingly, having flexible decomposer efficiency resulted in fewer behavioural parameter sets. This is surprising because it adds two extra parameters which should increase the possibility of finding better fits. It seems however that the two extra parameters decreased the probability of finding good parameter sets and because the calibration was run with the same number of trial parameter sets, this resulted in fewer behavioural parameter sets. 
The behaviour of $e_{0}$ points in the direction that decomposer efficiency might decrease with increasing temperature. This could be one of the explanations to why respiration is so strongly correlated to temperature. However, it can be difficult to compare $e_{0}$ between different models, between models and experiment, or indeed, between different experiments (cf. Devêvre and Horwáth, 2000; Steinweg et al., 2008). In experiments $e_{0}$ is not measured directly and a number of more or less explicit assumptions are introduced when calculating $e_{0}$ from measurable quantities such as consumed substrate and respiration; such assumptions may or may not distort the relation between conceptual and observed values. In models, we also simplify the system; simplifications that differ between models.

\subsubsection{Other temperature effects}

One part where the model has difficulty in reproducing the experiment is directly after temperature shifts, where respiration is underestimated and overestimated after shifts upward and downward, respectively. These deviations between predictions and observations are similar to those observed when extrapolating respiration rates obtained at constant temperatures to temperature shifts in the study by Wetterstedt et al. (2010). We propose that these deviations over a few days represent transient adjustments in decomposer properties to new conditions. A possible interpretation is that these transients result from decomposer adaptation to new temperatures and that previously cold-adapted organisms respond more strongly than previously warm-adopted (Bradford et al., 2008). It remains an open question how important such transients may be under field conditions where temperatures are changing continuously, albeit less rapidly than in most experimental studies.

It should also be born in mind that the temperature response we find in $e_{0}$ depends on the assumptions we have made about the temperature dependence of the other factors. For example, we are assuming that the dispersion function is temperature independent although the rate of decomposition is highly sensitive to the strength of dispersion (Hyvönen et al., 2005). This is a simplifying assumption but we are not aware of any experiments demonstrating temperature sensitivity of dispersion. Likewise, although there are theoretical arguments for the effects of quality on the temperature dependence of the rate of carbon utilisation (Bosatta and Ågren, 1999), this has not been tested rigorously experimentally. Allison et al. (2010) point out another complication from temperature dependent decomposer efficiency. If decomposer efficiency goes down with temperature, decomposers assimilating the same amount of carbon will produce less biomass, which in turn should lead to a lower production of extracellular enzymes that can release assimilable carbon. In our terminology this should correspond a positive coupling between $e_{0}$ and $u_{0}$. The increased loss of carbon caused by a temperature increase resulting from lowered decomposer ef- ficiency would then be counteracted by a lower use of carbon. Schimel and Weintraub (2003) suggest instead that lowered decomposer efficiency would not occur at the expense of enzyme production but rather lead to decreased microbial biomass. There is a possibility that different microbial populations are active at different temperatures and that cold-adaptation increases maintenance costs, i.e. decreases efficiency, which can lead to a negative coupling between growth rate and efficiency over changing temperatures (Lipson et al., 2009). In the laboratory experiment by Wetterstedt et al. (2010) the scope for changes in microbial populations was limited and the response should more reflect those of a fixed microbial composition although there were indications of changes in the microbial population (E. Bååth, unpublished data). The question of the mechanisms behind the temperature response of decomposition is still far from being solved and it is likely that we need to consider additional couplings between processes.

\section{Conclusions}

When fitting complex models with many adjustable parameters it is a common situation that many different parameter combinations are almost equally good and there is no additional information to be used for discriminating between them (Hyvönen et al., 2005). The strength of the GLUE method in a context like this one is that is does not select just one optimal set of parameters but allows all the possible parameters that match preselected conditions. What we learn in this study is that for almost all parameter combinations, $e_{0-5}$ is larger than $e_{0-15}$ and $e_{0-25}$. This is a strong suggestion that decomposer efficiency is, indeed, temperature dependent at least in the range $5-15^{\circ} \mathrm{C}$; above that range the results are less clear. This is a key result of this study.

A temperature-sensitive decomposer efficiency was shown to have a much stronger influence on quality differentiation, and thus respiration, than the temperature sensitivity of utilisation of different qualities. The difficulties in capturing changes in respiration rates at rapid temperature changes should caution us about extrapolating short term effects to longer time periods (cf. Wetterstedt et al., 2010); understanding the rate at which a microbial community can adjust requires more investigations. Our results show also that it is necessary to more carefully consider the temperature dependence of other processes than those directly coupled to the rate of substrate utilisation.

Acknowledgements. We would like to thank Keith Beven for help with the GLUE methodology.

Edited by: J.-A. Subke 


\section{References}

Ågren, G. I. and Bosatta, E.: Theoretical Ecosystem Ecology - Understanding Element Cycles, Cambridge University Press, Cambridge, 1998.

Ågren, G. I. and Wetterstedt, J. Å. M.: What determines the temperature response of soil organic matter decomposition?, Soil Biol. Biochem., 39(7), 1794-1798, 2007.

Allison, S. D., Wallenstein, M. D., and Bradford, M. A.: Soilcarbon response to warming dependent on microbial physiology, Nat. Geosci., 3, 336-340, 2010.

Beven, K.: A manifesto for the equifinality thesis, J. Hydrol., 320(1-2), 18-36, doi:10.1016/j.jhydrol.2005.07.007, 2006.

Beven, K.: Environmental modelling: An uncertain future?, Routledge, London, 2009.

Beven, K. and Binley, A.: The future of distributed models: Model calibration and uncertainty prediction, Hydrol. Process., 6(3), 278-298, doi:10.1002/hyp.3360060305, 1992.

Beven, K. J., Smith, P. J., and Freer, J. E.: So just why would a modeller choose to be incoherent? J. Hydrol., 354, 15-32, 2008.

Bosatta, E. and Ågren, G.: Soil organic matter quality interpreted thermodynamically, Soil Biol. Biochem., 31(13), 1889-1891, 1999.

Bosatta, E. and Ågren, G. I.: Exact solutions to the continuousquality equation for soil organic matter turnover, J. Theor. Biol., 224(1), 97-105, 2003.

Bradford, M. A., Davies, C. A., Frey, S. D., Maddox, T. R., Melillo, J. M., Mohan, J. E., Reynolds, J. F., Treseder, K. K., and Wallenstein, M. D.: Thermal adaptation of soil microbial respiration to elevated temperature, Ecol. Lett., 11(12), 1316-1327, doi:10.1111/j.1461-0248.2008.01251.x, 2008.

Bruun, S., Ågren, G. I., Christensen, B. T., and Jensen, L. S.: Measuring and modeling continuous quality distributions of soil organic matter, Biogeosciences, 7, 27-41, doi:10.5194/bg-7-272010, 2010.

Coleman, K. and Jenkinson, D. S.: RothC-26.3 - a model for the turnover of carbon in soil: Model description and users guide, Lawes Agricultural Trust, Harpenden, 1995.

Comins, H. N. and McMurtrie, R. E.: Long-term response of nutrient-limited forests to $\mathrm{CO}_{2}$ enrichment; Equilibrium behavior of plant-soil models, Ecol. Appl., 3(4), 666-681, 1993.
Davidson, E. A. and Janssens, I. A.: Temperature sensitivity of soil carbon decomposition and feedbacks to climate change, Nature, 440, 165-173, 7081, doi:10.1038/nature04514, 2006.

Devêvre, O. C. and Horwáth, W. R.: Decomposition of rice straw and microbial carbon use efficiency under different soil temperatures and moistures, Soil Biol. Biochem., 32(11-12), 1773-1785, doi:10.1016/S0038-0717(00)00096-1, 2000.

Hyvönen, R., Ågren, G. I., and Dalias, P.: Analysing temperature response of decomposition of organic matter, Glob. Change Biol., 11, 770-778, doi:10.1111/j.1365-2486.2005.00947.x, 2005.

Kirschbaum, M. U. F.: The temperature dependence of organicmatter decomposition-still a topic of debate, Soil Biol. Biochem., 38(9), 2510-2518, 2006.

Lipson, D. A., Monson, R. K., Schmidt, S. K., and Weintraub, M. N.: The trade-off between growth rate and yield in microbial communities and the consequences for under-snow soil respiration in a high elevation coniferous forest, Biogeochemistry, 95, 23-35, 2009.

Mantovan, P. and Todini, E.: Hydrological forecasting uncertainty assessment: Incoherence of the GLUE methodology, J. Hydrol., 330, 368-381, 2006.

Parton, W. J., Schimel, D. S., Cole, C. V., and Ojima, D. S.: Analysis of Factors Controlling Soil Organic Matter Levels in Great Plains Grasslands, Soil Sci. Soc. Am. J., 51(5), 1173-1179, 1987.

Schimel, J. P. and Weintraub, M. N.: The implications of exoenzyme activity on microbial carbon and nitrogen limitation in soil: a theoretical model, Soil Biol. Biochem., 35, 549-563, 2003.

Solomon, S.: Climate Change 2007: The physical science basis: Contribution of Working Group I to the Fourth Assessment Report of the Intergovernmental Panel on Climate Change, Cambridge University Press, Cambridge, 2007.

Steinweg, J. M., Plante, A. F., Conant, R. T., Paul, E. A., and Tanaka, D. L.: Patterns of substrate utilization during longterm incubations at different temperatures, Soil Biol. Biochem. 40(11), 2722-2728, 2008.

Wetterstedt, J. Å. M., Persson, T., and Ågren, G. I.: Temperature sensitivity and substrate quality in soil organic matter decomposition: results of an incubation study with three substrates, Glob. Change Biol., 16, 1806-1819, 2010. 